\title{
Los hábitos comunicativos y su influencia en la sofisticación política ciudadana
}

\author{
The influence of communicational habits on the \\ citizens' political sophistication
}

Carlos Muñiz / carlos.munizm@uanl.mx https://orcid.org/orcid.org/0000-0002-9021-8198

Universidad Autónoma de Nuevo León, México

Martín Echeverría / echevemartin@yahoo.com.mx https://orcid.org/orcid.org/0000-0001-6071-8725

Benemérita Universidad Autónoma de Puebla, México

Alejandra Rodríguez-Estrada / ale0323@gmail.com https://orcid.org/orcid.org/0000-0001-9963-2654

Benemérita Universidad Autónoma de Puebla, México

Oniel Francisco Díaz-Jiménez / oniel.diaz@ugto.mx https://orcid.org/orcid.org/0000-0002-2271-8940

Universidad de Guanajuato, México

\begin{abstract}
In this paper, an indicator to measure the level of electoral political sophistication was created to determine, along with the traditional indicator of political sophistication, the impact of communicative variables on their presence. For this, an analytical survey was applied after the elections of governors in the Mexican states of Nuevo Leon (2015), Puebla (2016) and Mexico (2017). Findings showed a positive impact of the follow-up of web pages regarding politics and interactive communication in both dimensions of sophistication as well electoral debates on electoral sophistication.
\end{abstract}

Key words: political sophistication, political knowledge, communicational habits, Internet, election campaign.

Resumen: En este artículo se creó un indicador para medir el grado de sofisticación política electoral con el fin de analizar, junto al indicador tradicional de sofisticación política, el impacto de las variables comunicativas en su nivel. Para ello, se trabajó con una encuesta analítica aplicada tras las elecciones a gobernador de los estados mexicanos de Nuevo León (2015), Puebla (2016) y Estado de México (2017). Los resultados mostraron un impacto positivo del seguimiento de páginas web de política y de la comunicación interactiva en ambas dimensiones de la sofisticación, y de los debates electorales en la sofisticación electoral.

Palabras clave: sofisticación política, conocimiento político, hábitos comunicativos, Internet, campaña electoral. 


\section{Introducción $^{1}$}

Una democracia sólida requiere instituciones estables y un sistema político asentado que permita la alternancia en el poder entre las diferentes opciones políticas, a través de la celebración de elecciones libres y justas. Si bien la existencia de estas estructuras es necesaria para lograr el anhelo de un sistema plenamente democrático, no es suficiente. Es habitual considerar una ciudadanía comprometida a nivel político y cívico como una de las condiciones para el buen funcionamiento de la democracia, para lo cual debe estar informada sobre los acontecimientos que se desarrollan en el terreno político del país (Buendía y Somuano, 2003; Delli-Carpini, 2004; Delli-Carpini y Keeter, 1996). En la medida en que los ciudadanos estén informados, serán capaces de expresar más correctamente sus juicios y articular de forma más apropiada sus preferencias políticas (Elenbaas et al., 2014).

La motivación o interés para adquirir información sobre política y la habilidad para procesarla y acumularla son elementos clave para la conformación del conocimiento político del ciudadano (Luskin, 1990; Zaller, 1992). Las repercusiones del conocimiento político mantenido por el ciudadano no sólo impactan en la existencia de una ciudadanía de calidad, sino también en la salud democrática sostenida por la sociedad en su conjunto (de Vreese y Boomgaarden, 2006). Ello ha motivado a que el proceso de generación del conocimiento político y sus repercusiones para el sistema haya sido objeto de estudio desde diferentes disciplinas. Es habitual encontrar en estos trabajos como intercambiables, o al menos muy relacionados, los conceptos de conocimiento político, conciencia política (political awareness) y sofisticación política (Popa, 2015; Rhee y Cappella, 1997; Zaller, 1992). Este último concepto se tomará como referencia en el presente estudio, al entenderse que puede englobar a los restantes.

Contar con individuos sofisticados en el terreno político es crucial para el buen desarrollo de una democracia, pues permite ejercer de manera más eficiente los derechos que como ciudadanos les corresponden. Ello se debe a que estas personas cuentan con un conocimiento más completo y exacto, basado en estructuras de conocimiento mentales sobre política más amplias y mejor organizadas e integradas (Luskin, 1987 y 1990; Rhee y Cappella, 1997). Esto les permite disponer y utilizar más y mejores recursos cognitivos para, por ejemplo, realizar una elección de voto bien razonado o participar

1 Este estudio fue financiado por el Programa para el Desarrollo Profesional Docente (Prodep) y el Consejo Nacional de Ciencia y Tecnología (Conacyt). 
en los diferentes terrenos que la democracia pone a su alcance (Buendía y Somuano, 2003; Hollander, 2014; Prior, 2005). Pero aunque una democracia ideal debería contar con una gran cantidad de ciudadanos altamente sofisticados, en la realidad es difícil que esto se produzca (Dassonneville, 2012). Normalmente, los ciudadanos menos sofisticados conforman una importante cantidad en la sociedad, e incluso no es raro que el ciudadano promedio muestre poco interés por lo que ocurre en el terreno político y que, por tanto, tampoco tenga una especial motivación para buscar información sobre política.

Ahora bien, aunque la ciudadanía esté motivada para acceder a la información (Dassonneville, 2012; Luskin, 1990), no siempre es sencillo obtenerla. De hecho, por lo general, el conocimiento político es adquirido de una forma vicaria a través de los medios (Elenbaas et al., 2014; Rhee y Cappella, 1997), donde discurren en buena medida las campañas y los debates políticos. El papel de los medios cobra, por tanto, un peso significativo, pues son mecanismos de traslación y traducción de la realidad política hacia la ciudadanía (Muñiz, 2012). Constituyen en gran medida la principal fuente informativa para gran parte de la sociedad (Cho et al., 2009; Rojas, 2006; Woolley et al., 2010), siendo su cobertura informativa de la política la moneda o herramienta con la cual operan las democracias (Gerth y Siegert, 2012). Por tanto, contribuyen a la gobernanza, pues permiten establecer un vínculo entre las opiniones de los individuos sobre los temas políticos y su decisión electoral (Henderson, 2014).

Quizás uno de los momentos políticos donde los medios adquieren un rol fundamental como mecanismo de información sea durante las campañas electorales. De hecho, se ha observado que durante éstas los ciudadanos amplían su conocimiento de los asuntos públicos (Craig et al., 2005; Hansen y Pedersen, 2014), lo cual contribuye a que realicen una elección informada.

Es posible que estas etapas de la vida política de los países conformen un escenario propicio para el incremento de la sofisticación política ciudadana, no tanto la vinculada con el nivel de conocimiento político factual (Weisberg y Nawara, 2010), sino más bien la relacionada con el conocimiento sobre propuestas de campaña, actitudes y competencia de los candidatos que concurren en la contienda electoral (Stevens, 2005). Aunque son abundantes los estudios sobre la sofisticación política vinculada al conocimiento factual (factual knowledge) de los ciudadanos, son menos los trabajos que han prestado atención a la sofisticación (Van Heerde et al., 2006) o a la conciencia electoral (Schuck et al., 2013) del votante, denominada en este trabajo como sofisticación política electoral. 
Atendiendo a estas premisas, el presente estudio buscó determinar el peso de las diferentes prácticas comunicativas realizadas por los ciudadanos - por ejemplo, la atención a contenidos de política, uso de páginas web de política, visionado de debates electorales, seguimiento de redes sociales y la conversación política- en la generación tanto de la sofisticación política factual como de la sofisticación política electoral. Para ello, se utilizaron los datos provenientes de encuestas aplicadas en los estados mexicanos de Nuevo León (2015), Puebla (2016) y Estado de México (2017), en el contexto de las campañas electorales a la gubernatura de cada una de dichas entidades.

\section{Literatura revisada}

\section{Conceptualización de la sofisticación politica}

Si bien el concepto de sofisticación política ha sido ampliamente utilizado en la literatura científica, tanto en disciplinas como la ciencia política, la psicología o la comunicación política, no existe uniformidad en cuanto a su definición conceptual (Popa, 2015). Como ya mencionaba Luskin (1987 y 1990), en gran medida la investigación elude el debate conceptual para centrarse en el terreno operacional de la medición de este concepto. A ello ha contribuido el hecho de que se haya conceptualizado desde dos disciplinas de forma diferente (Rhee y Cappella, 1997). Desde la ciencia política se suele revisar más la naturaleza ideológica del conocimiento político de las personas; mientras que en la psicología política se trabaja el concepto desde el proceso de construcción de conocimiento y su utilización por parte de los ciudadanos. Por ello, sus definiciones son múltiples. Algunas la vinculan con la confianza política de los ciudadanos, la cantidad de información que reciben, su interés por la política o su capacidad para realizar juicios políticos (Lee y Chang, 2011).

En su trabajo ampliamente citado, Luskin (1987: 860) define a la sofisticación política como el "número, diversidad y organización [tanto interna como inter-esquemática] de los esquemas políticos de una persona”. Es decir, la sofisticación se vincula con el nivel de "complejidad cognitiva" de la persona, entendiendo que cuanta mayor sea su experiencia política, mayor será su grado de sofisticación política y, por tanto, también su capacidad cognitiva (Lee y Chang, 2011; Zaller, 1992). En este sentido, en la medida en que alguien posea mayor sofisticación política, también tenderá a prestar más atención a los acontecimientos políticos, así como a tener una mayor capacidad 
para comprenderlos (Zaller, 1992: 21). Es decir, el ciudadano tendrá una mayor conciencia política (political awareness) por atender lo que ocurre en su entorno, lo cual contribuirá a aumentar su conocimiento político expresado en ideas objetivas y bien formadas acerca del sistema (Schuck et al., 2013).

Así pues, un ciudadano sofisticado es aquel que tiene "un compromiso intelectual o cognitivo [...] con los asuntos públicos" (de Vreese et al., 2011: 183); esto es, que cuenta con estructuras cognitivas o esquemas políticos complejos, profundos y organizados (Dassonneville, 2012; Miller, 2011; Stevens, 2005), los cuales se usan para procesar información, valorar a los actores políticos e identificar y articular sus intereses para comprometerse y participar en política de una manera más provechosa (Delli-Carpini y Keeter, 1996).

Estas estructuras presuponen el conocimiento político factual del ciudadano, entendido como las nociones acerca del sistema político, su estructura, funcionamiento y reglas de juego, y de sus principales actores protagonistas y actividades dentro del sistema, las cuales — frente a las opiniones subjetivas- pueden ser verificadas objetivamente (Delli Carpini y Keeter, 1996; Popa, 2015; Rhee y Cappella, 1997). Esta centralidad del conocimiento en la configuración de un ciudadano sofisticado ha llevado a que una parte de la literatura la haya utilizado como sinónimo de sofisticación (Buendía y Somuano, 2003; Delli-Carpini y Keeter, 1996), al señalar que es la única medida común en todos los estudios realizados (Rhee y Cappella, 1997).

Frente a esta concepción restringida de la sofisticación política que la vincula únicamente con una medida de conocimiento político, otros autores han planteado la necesidad de contar con más elementos para ofrecer una definición adecuada de lo que se espera de un ciudadano sofisticado (Miller, 2011; Muñiz, 2012). Así, por ejemplo, Guo y Moy (1998) señalan que la sofisticación también deriva del nivel de interés político que mantiene el ciudadano para adquirir la información. Esta idea también es planteada por autores como Catellani y Alberici (2012) o Dassonneville (2012: 27), quien señala que "las variables relacionadas con la sofisticación política [...] son 'los sospechosos habituales', conocimiento político e interés en la política”. Así, al igual que se pueden presentar diferentes niveles de conocimiento, también se puede presentar especialización y niveles en el interés hacia la política, contribuyendo a que no todos los ciudadanos se involucren por los mismos temas ni en la misma medida (Dalton, 2006; Norris, 2000). Por tanto, cuanto más se informe y reflexione un ciudadano sobre temáticas que considere de alto interés, más plausible es que también desarrolle mayores niveles de sofisticación política personal (Muñiz, 2012). 
En función de lo anteriormente dicho, se puede observar que para producirse un nivel significativo de sofisticación política en el ciudadano no sólo es necesario el mantenimiento de esquemas o estructuras mentales en forma de conocimiento político, sino también requiere de un nivel aceptable de interés que le motive a informarse de los asuntos públicos de su entorno (Buendía y Somuano, 2003; de Vreese et al., 2011; Smith y Durand, 1995). Esta conceptualización lleva a la sofisticación política al terreno de un constructo latente, en el cual se combinan rasgos como la atención a información política, el interés y el conocimiento político (Miller, 2011; Stevens, 2005). Por tanto, su operacionalización implicará la medición en un mismo concepto unidimensional del conocimiento factual, a partir de la suma de las respuestas correctas a diferentes cuestiones planteadas respecto de asuntos concretos, y el nivel de interés mantenido por el ciudadano a diferentes temas o asuntos vinculados con el contexto político (Catellani y Alberici, 2012; de Vreese et al., 2011; Guo y Moy, 1998; Miller, 2011; Muñiz, 2012; Popa, 2015; Singh y Roy, 2014; Stevens, 2005).

\section{La sofisticación politica en el contexto electoral}

La medición de la sofisticación política ha estado vinculada tradicionalmente al estudio del nivel de conocimiento político factual o real (factual knowledge) mantenido por el ciudadano (Luskin, 1987 y 1990; Miller, 2011; Rhee, 1997), normalmente a través de la comprobación de la cantidad de este conocimiento poseído (Rhee y Cappella, 1997). Sin embargo, este tipo de cálculo del conocimiento, más allá de realizar una verificación sobre el grado en el cual se está al corriente de una serie de hechos mediante preguntas factuales (factual questions), no llega a pulsar el verdadero nivel en que los ciudadanos procesan la información y construyen esquemas acerca de estos hechos que les permitan comprender su entorno político (de Vreese y Boomgaarden, 2006; Rhee y Cappella, 1997). Es decir, más allá del reconocimiento de instituciones, reglas y actores, es importante tener conciencia sobre cómo se posicionan los diferentes actores ante los asuntos de interés político y cómo se produce el debate en torno a los mismos (Henderson, 2014; Rhee y Cappella, 1997).

Si bien el conocimiento respecto al funcionamiento ordinario del sistema es necesario para la configuración de la ciudadanía, este se vuelve de crucial interés en el entorno de los contextos electorales. En ellos se requiere de ciudadanos que comprendan efectivamente la realidad política, a partir del conocimiento de las propuestas que se debaten en campaña (Weisberg 
y Nawara, 2010), para convertirse en votantes que toman decisiones y participan en el sistema (Delli-Carpini y Keeter, 1996; Henderson, 2014). Es sabido que las campañas electorales constituyen entornos donde el debate político tiende a aumentar entre los actores políticos, ya sean candidatos, partidos o sus representantes, y también se incrementa la interacción de los mismos con la ciudadanía (Drew y Weaver, 2006; Druckman y Leeper, 2012; Stevens, 2005). Aunque es cierto que en estos contextos se desarrollan estrategias para obtener beneficios electorales, contribuyendo a la activación y movilización de electorados (Claassen, 2011; Craig et al., 2005; Gerth y Siegert, 2012), también se genera un debate sobre los principales problemas de la política pública, lo cual coadyuva a educar y a actitudes y formas de pensar de los ciudadanos (Claassen, 2011; Weisberg y Nawara, 2010).

Se ha determinado ampliamente la existencia de una relación estrecha entre la posesión de un nivel aceptable de conocimiento político y la tendencia a una mayor participación (Buendía y Somuano, 2003; Norris, 2000; Rojas, 2006); algo que también se produce en el terreno electoral (Delli-Carpini y Keeter, 1996). Por lo general, los votantes que cuentan con mayor conocimiento político y, sobre todo, de los aspectos debatidos en campaña, tienden a comprometerse más y mejor, al emplear la información disponible durante las campañas electorales en la toma de su decisión final (Singh y Roy, 2014). Se deberían convertir, por tanto, en votantes educados y racionales, que seleccionan al partido o candidato que consideran más razonable en el proceso electoral tras reflexionar acerca de las diferentes propuestas presentadas por los mismos (Catellani y Alberici, 2012; Claassen, 2011; Dassonneville y Dejaeghere, 2014). Con todo, aspectos como una fuerte identificación partidaria o las actitudes políticas previas más desarrolladas, con que suelen contar los públicos más sofisticados, podrían moderar esta influencia de la campaña (Zaller, 1992).

La atención a lo acontecido en la campaña determina la existencia de ciudadanos con conciencia de campaña o electoral (campaign awareness), de forma similar a los ciudadanos que tienen mayor o menos conciencia política (political awareness). Por ello, ciertos autores entienden esta conciencia por los asuntos debatidos en campaña como la manifestación de una sofisticación política electoral (Schuck et al., 2013; Van Heerde et al., 2006). Se trata de un concepto multidimensional que implica medir el nivel de compromiso afectivo; es decir, el interés de los ciudadanos en el desarrollo de la campaña electoral, lo cual se ha detectado que tiende a que el reconocimiento de los aspectos debatidos aumente durante la misma (Hollander, 2005). Pero también el compromiso cognitivo, es decir, el nivel de conocimiento de 
campaña, frente al de conocimiento cívico, medido normalmente a través de preguntas sobre las posiciones políticas de los diferentes candidatos acerca de los asuntos debatidos en la contienda electoral o de sus propuestas programáticas específicas (Claassen, 2011; Hansen y Pedersen, 2014; Hollander, 2005; Rhee, 1997; Thorson, 2014; Van Heerde et al., 2006).

\section{Factores explicativos de la sofisticación política}

Es habitual encontrar en la literatura de disciplinas como la ciencia política o la comunicación estudios que han tratado de establecer los factores que inciden en el aumento o reducción del conocimiento político y la sofisticación política de los ciudadanos (Delli-Carpini y Keeter, 1996; Hollander, 2014; Luskin, 1990; Popa, 2015; Zaller, 1992). Los resultados de estas investigaciones plantean una amplia variedad de elementos que impactan en el interés y conocimiento político, y determinan el nivel de sofisticación política mantenida por el ciudadano. Ya desde su trabajo seminal, Luskin (1990) señalaba los tres principales factores que determinaban el nivel de sofisticación. En este sentido, se puede asumir que un ciudadano sofisticado es aquel que cuenta con habilidad cognitiva, es decir, herramientas para adquirir y procesar la información del entorno, así como oportunidad para poder adquirir esa información; factores que determinan qué tan fácilmente un ciudadano aprende sobre su entorno político (Elenbaas et al., 2014; Gordon y Segura, 1997).

Finalmente, Luskin (1990) plantea como un factor significativo la motivación o el deseo del ciudadano por aprender sobre lo que ocurre en su entorno. Este aspecto explica, en gran medida, que los individuos busquen y presten atención a la información, para comprender lo que sucede en el sistema. El hecho de contar con una motivación inherente para informarse, en combinación con una aceptable disponibilidad de información a través de fuentes como medios de comunicación, incide de forma clara en el proceso del aprendizaje del ciudadano, ayudando a configurar su conocimiento político (Elenbaas et al., 2014). Es por ello que regularmente la motivación ha sido asociada y operacionalizada como el nivel de interés que tiene el ciudadano en la política (Elenbaas et al., 2014; Popa, 2015). Si se asume esto, una falta de motivación supondría la primera barrera para que el proceso de adquirir información se produzca (Prior, 2005), y no tanto la falta de información y habilidades mantenidas por el sujeto para procesarla.

Ahora bien, aunque estas variables de motivación o habilidad puedan tener mayor impacto en la generación de sofisticación política que la relativa 
a la búsqueda de información (Luskin, 1990; Prior, 2005), no cabe duda de que es necesario que el ciudadano obtenga información para configurar su comprensión y conocimiento sobre lo que ocurre dentro del sistema. Dentro de esta búsqueda, Luskin (1990) incluyó el consumo de medios tradicionales, cuya influencia ha sido ampliamente estudiada.

En el caso de la prensa, se ha detectado constantemente que su consumo tiene un efecto positivo en el aumento de la sofisticación del ciudadano (Delli-Carpini y Keeter, 1996; Gordon y Segura, 1997; Guo y Moy, 1998; Hollander, 2005; Popa, 2015; Rhee y Cappella, 1997); y en el de la televisión, se ha observado que su consumo tiene una influencia negativa en la generación de conocimiento político (Gordon y Segura, 1997; Guo y Moy, 1998; Rhee y Cappella, 1997). En tanto, otros estudios han encontrado relaciones positivas del consumo televisivo (Hollander, 2005; Popa, 2015), debido principalmente al tipo de contenidos recibidos por este medio.

Se observa, por consiguiente, una diferencia entre la exposición a medios y la atención a contenidos concretos ofrecidos por parte de estos, como noticias, programas de política o debates electorales (de Vreese y Boomgaarden, 2006; Muñiz, 2012; Rojas, 2006). Al respecto, algunos autores señalan que la simple exposición genérica a medios es un indicador débil del aprendizaje político y conocimiento del ciudadano (Rhee y Cappella, 1997). Por ello, hay que prestar más atención a la exposición selectiva a contenidos de política, es decir, la atención a política en medios tradicionales. Esta variable ha sido detectada como un fuerte factor que explica el aumento de conocimiento político del ciudadano (Hollander, 2014; Rhee y Cappella, 1997; Rojas, 2006), incluso entre públicos más bien desmotivados en los cuales se puede producir un aprendizaje pasivo a partir de la exposición casual a estos contenidos (Prior, 2005). Este efecto positivo también ha sido percibido en contextos electorales, contribuyendo normalmente tanto la atención a política en medios tradicionales (de Vreese y Boomgaarden, 2006; Thorson, 2014), como la atención a los debates electorales realizados durante la campaña (Drew y Weaver, 2006; Druckman y Leeper, 2012) al aumento del conocimiento de los asuntos a debate y las características personales de los candidatos.

La llegada de los nuevos medios, en sus diferentes variantes, poco a poco está produciendo una migración del público de medios tradicionales a otros online (Woolley et al., 2010). Por ello, autores como Hollander (2014) recuerdan que en los nuevos estudios sobre cultura política es necesario incluir reactivos relativos a actividades como la lectura o consumo de noticias y contenidos online, ya que constituyen nuevos factores explicativos de las actitudes políticas del ciudadano. Así, varios autores señalan que los 
medios y redes sociales se están convirtiendo para buena parte de la sociedad en un medio crucial para el seguimiento de información política y, por tanto, para generar conocimiento político (Cho et al., 2009; Drew y Weaver, 2006; Hollander, 2014). Esto se debe a que su consumo no es tan demandante como otros medios tradicionales, lo cual posibilita al usuario de medios sociales buscar información más activa gracias a un proceso de comparación entre contenidos mediáticos; esto ha sugerido que puede generar un efecto nivelador del conocimiento político entre sus usuarios (Hollander, 2014; Prior, 2005). Dicho efecto positivo del consumo de contenidos de los nuevos medios sobre el conocimiento político también se ha detectado en el entorno de las campañas electorales (Drew y Weaver, 2006; Partheymüller y Faas, 2015; Woolley et al., 2010).

Finalmente, la literatura también ha sugerido un efecto importante sobre el conocimiento y sofisticación política de la variable relativa a la discusión o conversación política mantenida entre los ciudadanos, entendida como una variable crucial para el desarrollo político democrático (Cho et al., 2009; Rojas, 2006; Thorson, 2014). Cabe recordar que las prácticas comunicativas de los ciudadanos no se circunscriben al consumo mediático, sino también a los procesos de reflexión intra e interpersonal, generalmente entendidos como el diálogo político a través de la conversación, que pueden impactar logrando cambios en las actitudes políticas de los ciudadanos $(\mathrm{Mu}-$ ñiz, 2012). Gracias a estas estrategias de intercambio de información interpersonal, o incluso interactiva a través de plataformas digitales (Yamamoto et al., 2015), se puede incrementar el aprendizaje político, sirviendo esta conversación como un factor catalizador de la educación cívica deliberativa (Gastil y Dillard, 1999). Así lo pone de manifiesto, por ejemplo Thorson (2014), al encontrar que la exposición a noticias y la discusión política tenían un impacto positivo en el conocimiento de campaña mantenido por los ciudadanos.

A partir de los antecedentes empíricos revisados, se determinaron las siguientes preguntas de investigación respecto de la explicación de la sofisticación política factual y electoral:

PI1: ¿En qué medida la atención a política en medios tradicionales (a), el visionado de debates electorales (b), el uso de páginas web sobre política (c), el seguimiento de las redes sociales de Facebook y Twitter (d) y la conversación política interactiva (e) contribuyen al incremento de la sofisticación política factual?

PI2: ¿En qué medida la atención a política en medios tradicionales (a), el visionado de debates electorales (b), el uso de páginas web sobre política (c), el seguimiento de las redes sociales de Facebook y Twitter (d) y la conversación política interactiva (e) contribuyen al incremento de la sofisticación política electoral? 
Además, y teniendo en cuenta que se trata de un trabajo exploratorio comparado entre tres procesos electorales diferentes (vid infra), se planteó la siguiente pregunta de investigación aplicable a los modelos explicativos de la sofisticación política factual y electoral:

PI3: ¿Estas influencias eran iguales en los diferentes contextos electorales estudiados?

\section{Método seguido}

El presente estudio presenta un diseño metodológico explicativo bajo el método de encuesta analítica, la cual, antes que representar a amplios conjuntos poblacionales, pretende buscar asociaciones entre variables y sus patrones de comportamiento, determinar la direccionalidad de los mismos, probar hipótesis y generar hipótesis nuevas, entre otras finalidades, a la manera de los diseños experimentales, pero con controles estadísticos. Para ampliar el rango de escenarios analíticos y establecer un contraste entre ellos, se eligieron tres escenarios en donde recientemente se realizaron elecciones: los estados mexicanos de Nuevo León, Puebla y Estado de México, en los cuales se aplicó dicha encuesta analítica mediante una muestra de conveniencia de usuarios de redes sociales.

En este sentido, se invitó a los usuarios de Facebook de las tres entidades a participar en una encuesta online, promocionada como publicidad a través de esta red social. El trabajo de campo se hizo inmediatamente después de finalizar las elecciones a la gubernatura respectiva: durante los meses de junio y julio de 2015 para el caso de Nuevo León, tras la elección del 7 de junio; de junio a julio de 2016 para Puebla, tras la elección del 5 de junio; y de junio a julio de 2017 para el Estado de México, tras la elección del 4 de junio.

De los datos obtenidos, únicamente se contó con los relativos a los participantes que fueran mayores de edad ( $\geq 18$ años) y censados como votantes en los tres estados donde se realizó el estudio. Un $51.7 \%$ de la muestra de Nuevo León estaba constituido por mujeres $(n=152)$; en Puebla el $58.5 \%$ de la muestra $(n=176)$ fueron hombres; mientras que en el Estado de México el $50.4 \%$ eran mujeres $(n=180)$. Los participantes de Nuevo León tenían edades comprendidas entre 18 y 67 años $(M=34.06$, $D E=12.57)$, los de Puebla presentaron edades entre los 18 y 77 años $(M=$ $38.90, D E=14.59)$ y los del Estado de México oscilaban entre 18 y 71 años $(M=31.83, D E=12.51)$. 


\section{Medidas utilizadas}

Atención a política en medios tradicionales. Con la intención de conocer el nivel de consumo de contenidos sobre política en medios tradicionales durante las elecciones, se preguntó a los participantes qué tanto se informaron de la campaña a través de los siguientes contenidos: noticias en periódicos, noticias de la radio, noticias en la televisión, programas de política de la radio y programas de política en la televisión. Todos ellos se midieron con una escala de Likert que oscilaba entre nada (1) y mucho (5). La escala se creó a partir de la suma de los cinco ítems indicados, la cual contó además con consistencia interna buena, tanto para el caso de Nuevo León $(\alpha=.76)$, como para el de Puebla $(\alpha=.77)$ y Estado de México $(\alpha=.80)$.

Seguimiento de redes sociales durante la campaña. Los participantes en las encuestas fueron cuestionados acerca de en qué medida habían utilizado ciertas redes sociales para informarse de la campaña electoral a gobernador de cada uno de los estados. En concreto, se midió el seguimiento de Facebook y Twitter, a través de una escala de Likert, que oscilaba entre nada (1) y mucho (5), para cada una de las dos variables.

Conversación política interactiva. Se buscó detectar en qué medida los participantes habían mantenido conversaciones sobre política a través de la red. Para ello, se ocupó la escala que utilizaron en su estudio Yamamoto et al. (2015) y que está compuesta por cinco ítems que evalúan actividades como "escribir sobre asuntos políticos en la web" o "participar en discusiones políticas a través de la red”. Todos ellos fueron medidos mediante escalas de Likert de 5 puntos, entre nada (1) y mucho (5). La escala fue fiable para el caso de Nuevo León $(\alpha=.89)$, el de Puebla $(\alpha=.89)$ y el del Estado de México $(\alpha=.89)$.

Sofisticación politica factual. Con el objetivo de generar el indicador de sofisticación política factual, se tomaron como referencia varias propuestas realizadas por autores previamente, donde se recomienda unir las mediciones de interés y conocimiento político de los encuestados para generar un indicador de sofisticación política (Catellani y Alberici, 2012; Guo y Moy, 1998; Muñiz, 2012; Schuck et al., 2013; Singh y Roy, 2014; Stevens, 2005). Para ello, en primer lugar, se midió el interés hacia la política de los encuestados de los diferentes estados. Mediante escalas de Likert que oscilaban entre nada (1) y mucho (5), se preguntó por su interés hacia la política local o municipal, estatal, nacional o federal e internacional. La escala generada mediante la suma de estos reactivos presentó buena fiabilidad en el caso de Nuevo León $(\alpha=.72)$, Puebla $(\alpha=.83)$ y Estado de México $(\alpha=.71)$. 
En cuanto al conocimiento político factual, se formularon seis preguntas sobre la realidad política mexicana y estatal actual, determinando si los encuestados acertaban (1) o no (0) a preguntas como, por ejemplo: ¿Cuántos años dura el mandato del gobernador del estado? Con ambas variables se creó el indicador de sofisticación política, tomando como referencia la propuesta de de Vreese et al. (2011), de dar doble peso al componente cognitivo frente al afectivo, con la siguiente fórmula:

Sofisticación politica $=(($ Interés político $\times 1 / 2)+$ Conocimiento politico $) / 2$

El nuevo indicador contó con un rango teórico de variación que oscilaba entre 1.75 para reflejar la máxima sofisticación política y 0.25 para reflejar la mínima.

Sofisticación politica electoral. En relación con el nivel de sofisticación política sobre la contienda electoral demostrada por los encuestados, se siguió la misma propuesta que para el indicador anterior. Se midió el interés demostrado, en este caso, hacia las elecciones a la gubernatura de cada estado por los encuestados mediante una escala Likert de cinco puntos que oscilaba entre: estar nada interesado (1) y encontrarse muy interesado (5). Además, se midió el nivel de conocimiento político electoral mostrado por los participantes, mediante una batería de cinco preguntas, donde se pidió a los participantes que indicaran cuál candidato a la gubernatura había planteado cierta propuesta. Las respuestas fueron codificadas como: acierto (1) o fallo (0), con los mismos criterios seguidos para conocimiento político factual. Para crear el indicador se trabajó de igual manera que respecto a la sofisticación política factual.

Variables de control. Finalmente, se contempló el uso de una serie de variables de control, algunas de carácter sociodemográfico y otras de ámbito político. En concreto, se les cuestionó a los participantes acerca de su género ( 1 = masculino; 2 = femenino $)$ y su edad, en años cumplidos. También se pidió que indicaran cuál era, aproximadamente, el nivel de ingresos mensual de su núcleo familiar, con una escala que oscilaba entre menos de 6,000 pesos (1) y más de 30,001 pesos (4). También se evaluó el nivel de estudios de los participantes, con una escala que osciló entre: no tiene (1) y estudios de posgrado (7). Finalmente, se les pidió que se autoposicionaran en una escala ideológica que oscilaba entre: izquierda (0) y derecha (10). 


\section{Análisis de los resultados}

Para dar respuesta a las preguntas realizadas, se procedió a determinar los factores explicativos tanto de la sofisticación política factual como de la electoral, para lo cual se hicieron regresiones lineales múltiples jerárquicas para cada estado. Con esta técnica, que permite introducir las variables independientes en la ecuación en diferentes momentos, es posible generar modelos explicativos distintos y detectar las variaciones en el efecto producido por las diferentes variables predictoras sobre las variables criterio analizadas. En ambos casos, dentro del primer bloque se incorporaron las variables de control, constituidas por diferentes aspectos sociodemográficos medidos (modelo 1), e inmediatamente después se incorporaron las variables relativas a los hábitos comunicativos contemplados; es decir, la atención a política en medios tradicionales, el visionado de debates electorales, el consumo de páginas web sobre política, el seguimiento de redes sociales (Facebook y Twitter) y la conversación política interactiva (modelo 2). De esta manera se generó un control del impacto de las variables sociodemográficas, atendiendo a los resultados de falta de homogeneidad entre las muestras antes señaladas.

En cuanto al análisis sobre la sofisticación política factual (véase Tabla $1)^{2}$, las variables utilizadas en la regresión cumplieron el supuesto de independencia respecto de la variable dependiente, atendiendo a que el valor del test de Durbin-Watson fue aceptable en todas las muestras. Además, se descartaron problemas de colinealidad entre las variables usadas en cada ecuación. Entrando en los resultados, para el caso de Nuevo León se observó que tanto la atención a política en medios tradicionales $(\beta=.123, p=$ $.039)$, el visionado de debates electorales $(\beta=.142, p=.022)$, el seguimiento de Twitter $(\beta=.136, p=.026)$ y, especialmente, la conversación política interactiva durante la campaña electoral $(\beta=.246, p<.001)$ y el uso de páginas web sobre política $(\beta=.277, p<.001)$ contribuyeron a aumentar la sofisticación política.

Por su parte, en el caso de Puebla se observaron como explicativas las variables de conversación política interactiva $(\beta=.175, p=.009)$, la atención a política en medios tradicionales durante la campaña electoral $(\beta=.212, p<$ $.001)$ y, sobre todo, el uso de páginas web sobre política $(\beta=.267, p<.001)$. Finalmente, para el caso del Estado de México se detectó un impacto positivo del seguimiento de Twitter $(\beta=.123, p=.025)$, el uso de páginas web sobre política $(\beta=.200, p<.001) \mathrm{y}$, principalmente, la conversación política durante la campaña $(\beta=.208, p<.001)$.

2 Las tablas se encuentran al final del presente artículo, en el Anexo (Nota del editor). 
En tanto, el análisis de las variables explicativas de la sofisticación política electoral (véase Tabla 2) también cumplió con el supuesto de independencia respecto de la variable dependiente de todas las variables independientes en todas las muestras utilizadas, y tampoco se encontraron problemas de colinealidad, lo cual permitió utilizar todas las variables en los análisis realizados. En cuanto al peso que tuvieron las variables sobre hábitos comunicativos en la explicación de la sofisticación política electoral en el caso de Nuevo León, el análisis arrojó cuatro variables con un impacto estadísticamente significativo. En este sentido, el seguimiento de Twitter $(\beta=.176, p$ $=.003)$, el uso de páginas web sobre política $(\beta=.218, p<.001)$, la conversación política interactiva $(\beta=.252, p<.001)$ y el visionado de debates electorales $(\beta=.292, p<.001)$ lograron impactar de una forma consistente en el incremento de la misma.

Por su parte, en el caso de Puebla, la ecuación arrojó tres variables explicativas, pues el seguimiento de Facebook durante la campaña electoral $(\beta=$ $.161, p=.015)$, el visionado de debates $(\beta=.212, p<.001)$ y el uso de páginas web $(\beta=.299, p<.001)$ contribuyeron a la mejora de la sofisticación electoral de los participantes. Por último, durante la campaña electoral del Estado de México, el visionado de debates $(\beta=.178, p=.002)$, el uso de páginas web $(\beta=.222, p<.001)$ y la conversación política interactiva $(\beta$ $=.207, p<.001)$ coadyuvaron positivamente al incremento de la sofisticación electoral de los participantes.

\section{Discusión y conclusiones}

El objetivo principal de este estudio fue determinar en qué medida cada una de las prácticas comunicativas contempladas en el trabajo impactaban en la determinación o no de la sofisticación política, tanto factual como electoral, de los ciudadanos. En concreto, se pretendía evaluar la influencia ejercida por la atención a contenidos de política en medios tradicionales, el visionado de debates electorales, el uso de páginas web sobre política, el seguimiento de las redes sociales Facebook y Twitter, y la conversación política interactiva, en el contexto de las campañas electorales a gobernador de los estados mexicanos de Nuevo León (2015), Puebla (2016) y Estado de México (2017). Atendiendo a este planteamiento, la primera pregunta se centró en el análisis de los factores explicativos de la sofisticación política factual, que deriva del nivel de interés hacia la política y conocimiento sobre el sistema político sostenido por los ciudadanos. 
Los resultados vislumbran un decrecimiento en el impacto de la atención a contenidos de política a través de medios tradicionales, una variable que, sin embargo, había sido habitualmente una de las principales determinantes del conocimiento y sofisticación política de los ciudadanos (Hollander, 2014; Muñiz, 2012; Prior, 2005; Rhee y Cappella, 1997; Rojas, 2006). Si bien en la mayoría de las elecciones, salvo en el Estado de México, el seguimiento de estos contenidos seguía contribuyendo a aumentar la sofisticación política, se observa que otras variables comunicativas, relacionadas con el entorno digital, comienzan a posicionarse como altamente explicativas de este compromiso cognitivo del ciudadano.

Tanto el seguimiento de páginas web sobre política, como las manifestaciones de conversación política a través de plataformas en Internet, coadyuvaron de forma significativa a conseguir ciudadanos más sofisticados en el terreno del conocimiento factual. Por tanto, se comprueban hipótesis positivas sobre el impacto de los medios sociales en el conocimiento político ya planteadas por autores previamente (Cho et al., 2009; Hollander, 2014; Woolley et al., 2010).

Respecto a la segunda pregunta de investigación en este trabajo, se observa que eran tres las variables comunicativas que tienen una mayor probabilidad de determinar la sofisticación política mantenida por los ciudadanos en el ámbito electoral. En este sentido, en todas las campañas electorales estudiadas la conversación política en el ámbito digital, el consumo de páginas web sobre política y el visionado de debates electorales parecen ser los factores que contribuyen a que los ciudadanos incrementen su interés por lo que ocurre en la campaña y desarrollen mayor conocimiento sobre las propuestas realizadas por los candidatos.

Por lo tanto, de nuevo parece decaer la influencia del consumo de contenidos en medios tradicionales, de forma contraria a lo que ciertos autores han encontrado en estudios precedentes (de Vreese y Boomgaarden, 2006; Thorson, 2014). Sin embargo, se confirman las hipótesis planteadas acerca de la influencia positiva del debate, en tanto que espacio tradicional de campaña, y las de los nuevos medios, propuestas por autores previamente (Drew y Weaver, 2006; Druckman y Leeper, 2012; Partheymüller y Faas, 2015; Woolley et al., 2010).

Junto al estudio de elementos explicativos de la sofisticación política, tanto factual como electoral, también se planteó en la tercera pregunta de investigación si los modelos explicativos que se obtuvieran variarían en función del contexto electoral estudiado. Los resultados ponen de manifiesto patrones bastante similares entre las tres muestras utilizadas en relación con 
la explicación de la sofisticación política factual, a pesar de corresponder a estados y años diferentes.

En los tres casos se presentaron como principales variables explicativas las relativas al consumo de páginas web y la conversación política interactiva, siendo alta también la influencia de la atención a medios tradicionales. En cuanto al modelo explicativo de la sofisticación política electoral, también se encuentra un patrón muy determinado de impacto de ciertas variables comunicativas. El consumo de páginas web y la conversación interactiva nuevamente surgen, incorporándose en este caso el visionado de debates, como elementos transversales de explicación en todos los estados. Sólo destacan ligeras diferencias entre estos respecto al uso de algunas de las redes sociales contempladas en ambos tipos de sofisticación.

Los resultados del estudio ponen de manifiesto la importancia que están comenzando a tener Internet y los medios sociales en la determinación de las actitudes políticas, entre ellas la sofisticación política. Si bien no se puede obviar el papel que los medios tradicionales siguen jugando en el aprendizaje político de los ciudadanos, es cierto que cada vez una mayor parte de la sociedad comienza a utilizar las diferentes plataformas digitales para informarse de política (Drew y Weaver, 2006), en especial durante los procesos electorales (Partheymüller y Faas, 2015; Woolley et al., 2010). Por tanto, este parece ser el nuevo escenario donde buena parte de los ciudadanos se convierten en los procesadores profundos de contenidos políticos que señalaban autores como Luskin (1990) o Prior (2005). Así, los resultados de este estudio posibilitan asumir que buena parte de los altamente sofisticados están obteniendo información a través de páginas webs sobre política, y posteriormente sometiendo a reflexión esta información mediante estrategias de conversación política dentro de la propia red.

En efecto, Internet ha abierto una importante oportunidad a los diferentes actores políticos para diseminar su discurso político, asumiendo que en los nuevos medios la frontera entre productor y consumidor se rompe, y los discursos de los diferentes actores están altamente interrelacionados entre sí. Aunque los medios tradicionales sigan siendo fuentes primarias de información, y en especial la televisión sigue siendo un medio muy consumido, Internet comienza a ser un mecanismo altamente efectivo para trasladar información política e impactar en el conocimiento de la ciudadanía.

En especial entre el segmento más joven de la población, mucho más vinculado con el uso de los medios y redes sociales, para quienes buena parte de la información sobre elecciones llega a través de Internet (Woolley et al., 2010). Por eso es necesario seguir indagando sobre el acceso a la política a 
través de Internet por esta parte de la población, pues es probable que en este grupo etario el impacto del consumo de redes sociales sea más explícito que lo encontrado en el presente estudio para población general.

Se ha planteado ampliamente por parte de los académicos que aquellos más sofisticados tienden a ser menos tendentes al cambio de opiniones por parte de los medios, y son menos influenciables por los anuncios de campaña, pues cuentan con redes asociativas de información más ricas que los poco sofisticados (Lee y Chang, 2011; Miller, 2011; Zaller, 1992). Este tipo de hipótesis llevan a la duda acerca de los resultados obtenidos en el estudio, en el sentido de si el consumo de política por Internet está rompiendo esta barrera entre altos y bajos sofisticados, en un proceso de nivelación como el planteado por Hollander (2014) o Prior (2005). En este sentido, es necesario continuar indagando sobre las diferencias presentadas en esta nueva ciudadanía digital, para determinar hasta qué punto los medios están incidiendo en la forma como se produce el aprendizaje político, así como en la determinación de los niveles de sofisticación política mantenidos por la ciudadanía.

\section{Referencias}

Buendía, Jorge y Fernanda Somuano (2003), "Participación electoral en nuevas democracias: la elección presidencial de 2000 en México", en Política y Gobierno, vol. 10, núm. 2, México: Centro de Investigación y Docencia Económicas (CIDE).

Catellani, Patrizia y Alberici, Augusta (2012), "Does the candidate matter? Comparing the voting choice of early and late deciders", en Political Psychology, vol. 33, núm. 5, USA: Wiley. Disponible en: https://doi.org/10.1111/j.1467-9221.2012.00891.x [15 de septiembre de 2014].

Cho, Jaeho et al. (2009), "Campaigns, reflection, and deliberation: Advancing an O-S-RO-R model of communication effects", en Communication Theory, vol. 19, núm. 1, USA: Wiley. Disponible en: https://doi.org/10.1111/j.1468-2885.2008.01333.x [11 de febrero de 2010].

Claassen, Ryan (2011), "Political awareness and electoral campaigns: Maximum effects for minimum citizens?", en Political Behavior, vol. 33, núm. 2, USA: Springer. Disponible en: https://doi.org/10.1007/s11109-010-9129-6 [2 de febrero de 2017].

Craig, Stephen $e t$ al. (2005), "Issue-related learning in a gubernatorial campaign: a panel study", en Political Communication, vol. 22, núm. 4, USA: Taylor \& Francis. Disponible en: https://doi.org/10.1080/10584600500311501 [2 de febrero de 2017].

Dalton, Russel (2006), Citizen politics: Public opinion and political parties in advanced industrial democracies, USA: CQ Press.

Dassonneville, Ruth (2012), "Electoral volatility, political sophistication, trust and efficacy: A study on changes in voter preferences during the Belgian regional elections of 2009", en Acta Politica, vol. 47, núm. 1, Reino Unido: Palgrave. Disponible en: https://doi. org/10.1057/ap.2011.19 [10 de octubre de 2013]. 
Carlos Muñiz, Martín Echeverría, Alejandra Rodríguez-Estrada y Oniel Francisco Díaz-Jiménez. Los hábitos comunicativos y su influencia en la sofisticación politica ciudadana

Dassonneville, Ruth y Dejaeghere, Yves (2014), "Bridging the ideological space: A crossnational analysis of the distance of party switching", en European Journal of Political Research, vol. 53, núm. 3, USA: Wiley. Disponible en: https://doi.org/10.1111/14756765.12049 [ 15 de septiembre de 2014].

de Vreese, Claes y Boomgaarden, Hajo (2006), "News, political knowledge and participation: the differential effects of news media exposure on political knowledge and participation”, en Acta Politica, vol. 41, núm. 4, Reino Unido: Palgrave. Disponible en: https://doi.org/10.1057/palgrave.ap.5500164 [2 de febrero de 2017].

de Vreese, Claes et al. (2011), "(In)direct framing effects: The effects of news media framing on public support for Turkish membership in the European Union”, en Communication Research, vol. 38, núm. 2, USA: Sage. Disponible en: https://doi. org/10.1177/0093650210384934 [2 de febrero de 2017].

Delli-Carpini, Michael (2004), "Mediating democratic engagement: The impact of communications on citizens' involvement in political and civic life", en Kaid, L. [ed.], Handbook of political communication, USA: Lawrence Erlbaum.

Delli-Carpini, Michael y Keeter, Scott (1996), What Americans know about politics and why it matters, USA: Yale University Press.

Drew, Dan y Weaver, David (2006), "Voter learning in the 2004 presidential election: Did the media matter?", en Journalism \& Mass Communication Quarterly, vol. 83, núm. 1, USA: Sage. Disponible en: https://doi.org/10.1177/107769900608300103 [2 de febrero de 2017].

Druckman, James y Leeper, Thomas (2012), "Learning more from political communication experiments: Pretreatment and its effects", en American Journal of Political Science, vol. 56, núm. 4, USA: Wiley. Disponible en: https://doi.org/10.1111/j.15405907.2012.00582.x [2 de febrero de 2017].

Elenbaas, Matthijs et al. (2014), "Reconciling passive and motivated learning: The saturationconditional impact of media coverage and motivation on political information", en Communication Research, vol. 41, núm. 4, USA: Sage. Disponible en: https://doi. org/10.1177/0093650212467032 [25 de abril de 2014].

Gastil, John y Dillard, James (1999), "Increasing political sophistication through public deliberation”, en Political Communication, vol. 16, núm. 1, USA: Taylor \& Francis. Disponible en: https://doi.org/10.1080/105846099198749 [2 de febrero de 2017].

Gerth, Matthias y Siegert, Gabriele (2012), "Patterns of consistence and constriction: How news media frame the coverage of direct democratic campaigns", en American Behavioral Scientist, vol. 56, núm. 3, USA: Sage. Disponible en: https://doi. org/10.1177/0002764211426326 [30 de abril de 2012].

Gordon, Stacy y Segura, Gary (1997), "Cross-national variation in the political sophistication of individuals: Capability or choice?”, en The Journal of Politics, vol. 59, núm. 1, USA: Chicago University Press. Disponible en: https://doi.org/10.2307/2998218 [10 de noviembre de 2013].

Guo, Zhongshi y Moy, Patricia (1998), "Medium or message? Predicting dimensions of political sophistication”, en International Journal of Public Opinion Research, vol. 10, núm. 1, Reino Unido: Oxford University Press. Disponible en: https://doi. org/10.1093/ijpor/10.1.25 [24 de abril de 2015].

Hansen, Kasper y Pedersen, Rasmus (2014), "Campaigns matter: How voters become knowledgeable and efficacious during election campaigns”, en Political Communication, 
Convergencia Revista de Ciencias Sociales, núm. 77, 2018, Universidad Autónoma del Estado de México

vol. 31, núm. 2, USA: Taylor \& Francis. Disponible en: https://doi.org/10.1080/1058 4609.2013.815296 [23 de octubre de 2014].

Henderson, Michael (2014), "Issue publics, campaigns, and political knowledge", en Political Behavior, vol. 36, núm. 3, USA: Springer. Disponible en: https://doi.org/10.1007/ s11109-013-9243-3 [2 de febrero de 2017].

Hollander, Barry (2005), "Late-night learning: Do entertainment programs increase political campaign knowledge for young viewers?", en Journal of Broadcasting \& Electronic Media, vol. 49, núm. 4, Reino Unido: Routledge Journals. Disponible en: https://doi.org/10.1207/s15506878jobem4904_3 [6 de octubre de 2014].

Hollander, Barry (2014), "The role of media use in the recall versus recognition of political knowledge”, en Journal of Broadcasting \& Electronic Media, vol. 58, núm. 1, Reino Unido: Routledge Journals. Disponible en: https://doi.org/10.1080/08838151.201 3.875019 [ 5 de octubre de 2014].

Lee, Yu-Kang y Chang, Chun-Tuan (2011), "Missing ingredients in political advertising: The right formula for political sophistication and candidate credibility", en The Social Science Journal, vol. 48, núm. 4, Holanda: Elsevier Science. Disponible en: https://doi. org/10.1016/J.SOSCIJ.2011.04.001 [29 de octubre de 2013].

Luskin, Robert (1987), "Measuring political sophistication", en American Journal of Political Science, vol.31, núm.4, USA: Wiley. Disponible en: https://doi.org/10.2307/2111227 [2 de febrero de 2017].

Luskin, Robert (1990), "Explaining political sophistication", en Political Behavior, vol. 12, núm. 4, USA: Springer. Disponible en: https://doi.org/10.1007/BF00992793 [2 de febrero de 2017].

Miller, Patrick (2011), “The Emotional citizen: Emotion as a function of political sophistication”, en Political Psychology, vol. 32, núm. 4, USA: Wiley. Disponible en: https://doi.org/10.1111/j.1467-9221.2011.00824.x [15 de septiembre de 2014].

Muñiz, Carlos (2012), "Creando ciudadanos comprometidos. Aportación de los hábitos comunicativos al desarrollo de la sofisticación política entre los jóvenes”, en Revista Mexicana de Opinión Pública, núm. 12, México: Universidad Nacional Autónoma de México.

Norris, Pippa (2000), A virtuous circle: Political communication in post-industrial democracies, Reino Unido: Oxford University Press.

Partheymüller, Julia y Faas, Thorsten (2015), “The impact of online versus offline campaign information on citizens' knowledge, attitudes and political behaviour: Comparing the german federal elections of 2005 and 2009", en German Politics, vol. 24, núm. 4, Reino Unido: Routledge Journals. Disponible en: https://doi.org/10.1080/09644008.2015 .1021789 [8 de octrubre de 2015].

Popa, Sebastian (2015), "Political sophistication in Central and Eastern Europe", en Party Politics, vol. 21, núm. 3, Inglaterra: Sage. Disponible en: https://doi. org/10.1177/1354068813487104 [5 de octubre de 2014].

Prior, Markus (2005), "News vs. entertainment: How increasing media choice widens gaps in political knowledge and turnout", en American Journal of Political Science, vol. 49, núm. 3, USA: Wiley. Disponible en: https://doi.org/10.1111/j.1540-5907.2005.00143.x [2 de septiembre de 2010].

Rhee, June (1997), "Strategy and issue frames in election campaign coverage: A social cognitive account of framing effects”, en Journal of Communication, vol. 47, núm. 3, 
Carlos Muñiz, Martín Echeverría, Alejandra Rodríguez-Estrada y Oniel Francisco Díaz-Jiménez. Los hábitos comunicativos y su influencia en la sofisticación politica ciudadana

USA: Wiley. Disponible en: https://doi.org/10.1111/j.1460-2466.1997.tb02715.x [31 de julio de 2006].

Rhee, June y Cappella, Joseph (1997), “The role of political sophistication in learning from news”, en Communication Research, vol. 24, núm. 3, USA: Sage. Disponible en: https://doi.org/10.1177/009365097024003001 [31 de julio de 2016].

Rojas, Hernando (2006), "Comunicación, participación y democracia”, en Universitas Humanistica, núm. 62, Colombia: Pontificia Universidad Javeriana.

Schuck, Andreas et al. (2013), "Cynics all around? The impact of election news on political cynicism in comparative perspective", en Journal of Communication, vol. 63, núm. 2, USA: Wiley. Disponible en: https://doi.org/10.1111/jcom.12023 [11 de abril de 2014].

Singh, Shane P. y Roy, Jason (2014), "Political knowledge, the decision calculus, and proximity voting”, en Electoral Studies, vol. 34, Reino Unido: Elsevier. Disponible en: https://doi.org/10.1016/j.electstud.2013.11.007 [15 de septiembre de 2014].

Smith, Ma. Marcia y Durand, Víctor Manuel (1995), "La acción colectiva y su papel contradictorio en la construcción de la ciudadanía en México", en Estudios Sociológicos, vol. 13, núm. 38, México: El Colegio de México.

Stevens, Daniel (2005), "Separate and unequal effects: Information, political sophistication and negative advertising in American elections”, en Political Research Quarterly, vol. 58, núm. 3, USA: Sage. Disponible en: https://doi.org/10.1177/10659129050580 0304 [5 de octubre de 2014].

Thorson, Emily (2014), "Beyond opinion leaders. How attempts to persuade foster political awareness and campaign learning”, en Communication Research, vol. 41, núm. 3, USA: Sage. Disponible en: https://doi.org/10.1177/0093650212443824 [5 de octubre de 2014].

Van Heerde, Jennifer et al. (2006), "Barriers to participation, voter sophistication and candidate spending choices in US senate elections", en British Journal of Political Science, vol. 36, núm. 4, USA: Cambridge University Press. Disponible en: https:// doi.org/10.1017/S0007123406000391 [2 de febrero de 2017].

Weisberg, Herbert y Nawara, Steven (2010), "How sophistication affected the 2000 presidential vote: Traditional sophistication measures versus conceptualization", en Political Behavior, vol. 32, núm. 4, USA: Springer. Disponible en: https://doi. org/10.1007/s11109-010-9117-x [10 de octubre de 2013].

Woolley, Julia et al. (2010), "The 2008 presidential election, 2.0: A content analysis of usergenerated political facebook groups", en Mass Communication and Society, vol. 13, núm. 5, Reino Unido: Routledge Journals. Disponible en: https://doi.org/10.1080/ 15205436.2010.516864 [29 de junio de 2013].

Yamamoto, Masahiro et al. (2015), "Social media and mobiles as political mobilization forces for young adults: Examining the moderating role of online political expression in political participation”, en New Media \& Society, vol. 17, núm. 6, Inglaterra: Sage. Disponible en: https://doi.org/10.1177/1461444813518390 [2 de febrero de 2017].

Zaller, John (1992), The nature and origins of mass opinion, USA: Cambridge University Press. 


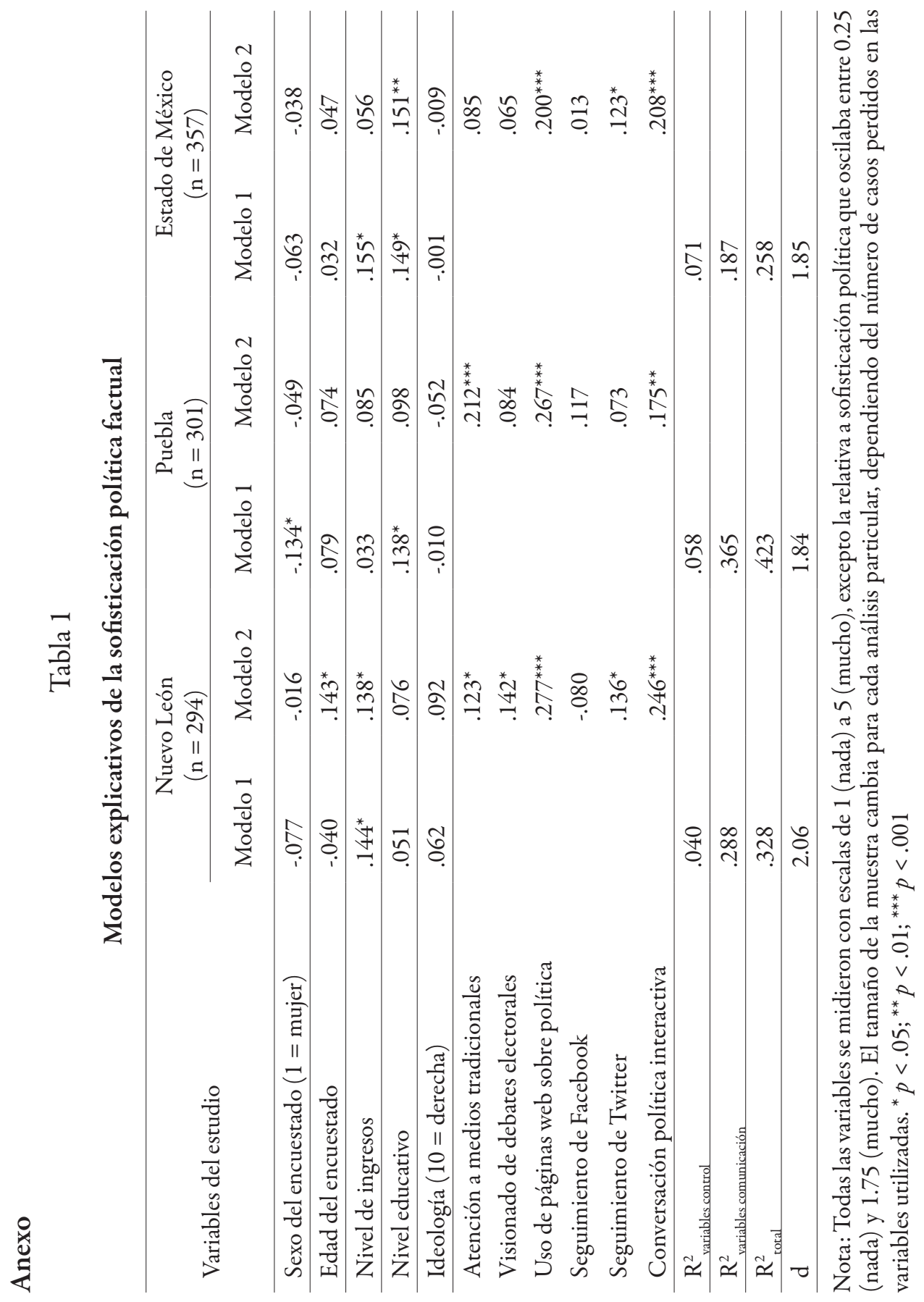


Carlos Muñiz, Martín Echeverría, Alejandra Rodríguez-Estrada y Oniel Francisco Díaz-Jiménez. Los hábitos comunicativos y su influencia en la sofisticación politica ciudadana

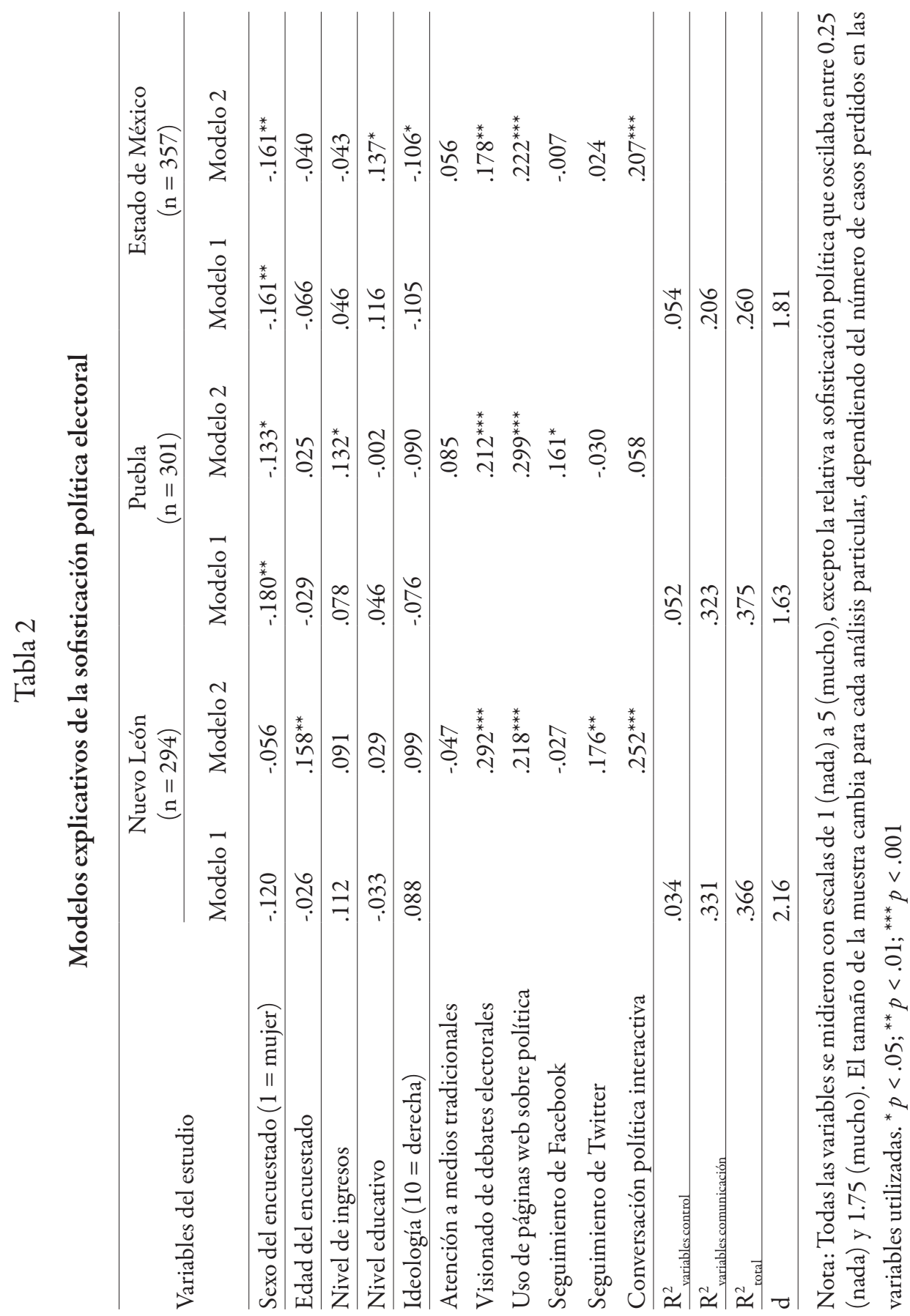


Carlos Muñiz. Doctor en Comunicación. Profesor Titular en la Facultad de Ciencias Políticas y Relaciones Internacionales de la Universidad Autónoma de Nuevo León. Líneas de investigación: efectos mediáticos en el área de la comunicación política, tratamiento informativo (framing) de los acontecimientos. Publicaciones recientes: "Framing of the electoral processes: the stages of the campaign as a moderator of the presence of the political frames in the news", en Palabra Clave (en prensa); "Engagement of politicians and citizens in the cyber campaign on Facebook: A comparative analysis between Mexico and Spain", en Contemporary Social Science (en prensa); "Political sophistication as a mediator in the relation between media consumption and citizen participation. Evidence from the O-S-R-O-R model", en Communication \& Society, vol. 30, núm. 3 (2017).

Martín Echeverría. Doctor en Comunicación y Cultura. Profesor Investigador del Instituto de Ciencias de Gobierno y Desarrollo Estratégico, Universidad Benemérita Autónoma de Puebla. Líneas de investigación: infoentretenimiento en la cobertura periodística de las elecciones y el desempeño de los debates presidenciales. Publicaciones recientes: "Sesgo partidista en medios informativos. Una crítica metodológica y propuesta”, en Comunicación y Sociedad, vol. 30 núm. 22 (2017); "Personalización política e infoentretenimiento periodístico. Un estudio desde los encuadres", en Cuadernos.info, núm. 41 (2017); "Infoentretenimiento periodístico en la cobertura de las elecciones. El caso de los debates presidenciales", en Convergencia Revista de Ciencias Sociales, núm. 74 (2017).

Alejandra Rodríguez-Estrada. Doctora en Ciencias Sociales. Becaria posdoctoral Conacyt en el Instituto de Ciencias de Gobierno y Desarrollo Estratégico, Universidad Autónoma de Puebla. Líneas de investigación: estudio de la comunicación política como campo científico, la socialización política y la participación política. Publicaciones recientes: "La tensión centro-periferia en la producción del campo de la comunicación política. El caso mexicano", en Revista Latina de Comunicación Social, núm. 72 (2017); "Tensiones teóricas en torno al estudio de la ciencia. De la sociología de la ciencia al concepto de campo científico", en Andamios, vol. 13 núm. 31 (2016); "Prácticas objetivadas y subjetivadas en la producción de investigadores del campo científico de la comunicación política en México", en Global Media Journal, vol. 13, núm. 25 (2016). 
Oniel Francisco Díaz-Jiménez. Doctor en Ciencia Política y Estudios Internacionales. Profesor-investigador en el Departamento de Estudios Políticos y de Gobierno, Universidad de Guanajuato. Líneas de investigación: gobierno y política comparada, elecciones, partidos políticos y sistemas de partidos, comunicación política y opinión pública. Publicaciones recientes: "Los efectos de la comunicación política en el compromiso político de los jóvenes en la elección presidencial mexicana de 2012", en Revista Mexicana de Ciencias Politicas y Sociales, vol. 62, núm. 229 (2017); "Election campaigns, the media and their impact on civic engagement of Mexicans in the 2012 presidential election", en Comunicación y Sociedad, núm. 29 (2017); "Las redes sociales en las campañas de los candidatos a diputados locales del PRI, el PAN y el PRD en las elecciones de 2015 en el Estado de México", en Apuntes Electorales, vol. 16, núm. 57 (2017).

Recepción: 13 de diciembre de 2017.

Aprobación: 12 de febrero de 2018. 
